\title{
Влияние фазового перехода второго рода на электропроводность структуры металл/полупроводник
}

\author{
(С) И.Р. Набиуллин ${ }^{1}$, Р.М. Гадиев ${ }^{1, \uparrow}$, А.Н. Лачинов ${ }^{2}$ \\ ${ }^{1}$ Башкирский государственный педагогический университет им. М. Акмуллы, \\ 450000 Уфра, Россия \\ ${ }^{2}$ Институт фризики молекул и кристаллов Уфримского научного центра Российской академии наук, \\ 450054 Уфра, Россия \\ I E-mail: gadiev.radik@gmail.com \\ (Поступила в Редакцию 15 ноября 2018 г. \\ В окончательной редакции 26 ноября 2018 г. \\ Принята к публикации 26 ноября 2018 г.)
}

\begin{abstract}
Представлены результаты исследования свойств потенциального барьера $\mathrm{Cr}-p$-Si вблизи температур фазового перехода антиферромагнетик/парамагнетик в Cr. Обнаружено значительное изменение потенциального барьера и аномальное увеличение проводимости в структуре $\mathrm{Cr}-p$ - $\mathrm{Si}-\mathrm{Au}$ вблизи температуры фазового перехода 2-го рода антиферромагнетик/парамагнетик в хроме. Установлено, что по мере приближения к температуре фазового перехода в структуре наблюдается нарастание флуктуаций тока. Интерпретация полученных экспериментальных результатов строится на предположении о том, что наблюдаемые изменения электронных транспортных свойств контакта $\mathrm{Cr}-\mathrm{Si}$ обусловлены изменением положения квазиуровня Ферми хрома в результате фазового перехода 2-го рода.
\end{abstract}

DOI: 10.21883/FTP.2019.04.47439.9021

\section{1. Введение}

Современные электронные устройства в своей основе используют различные свойства потенциальных барьеров, сформированных на границах раздела металл/полупроводник [1]. Задача обеспечения стабильности работы устройств в заданном температурном интервале предполагает либо отсутствие, либо слабую зависимость параметров барьера от температуры [2]. В то же время одним из факторов, определяющих свойства потенциального барьера, является работа выхода металла $\varphi_{M}$, связанная известным соотношением с положением уровня Ферми металла: $\varphi_{M}=E_{\mathrm{F} M}-E_{\mathrm{vak}}$ $\left(E_{\mathrm{vak}}-\right.$ энергия уровня вакуума). Как было показано в работе [3], на положение уровня Ферми должны влиять не только энергетическое распределение электронов, но и структура кристалла. Из чего следует, что при изменении положения уровня Ферми металла, например, в области его фазового перехода, будет изменяться высота потенциального барьера, определяемая в общем случае как $\varphi=\varphi_{M}-\xi_{s m}$, где $\xi_{s m}-$ энергия сродства к электрону полупроводника. При этом в зависимости от того увеличивается или уменьшается потенциальный барьер можно определить относительное изменение положения уровня Ферми и направление этого изменения относительно равновесного состояния.

Ранее подобный подход был использован для интерпретации результатов экспериментов по изменению электропроводности в МДМ структуре, содержащей тонкую полимерную прослойку в качестве диэлектрика между двумя разными металлами. В одном из металлов инициировался фазовый переход I или II рода $[4,5]$. Вблизи температуры фазового перехода регистриро- валось существенное изменение электропроводности структуры. Однако наличие органического диэлектрика, электронные свойства которого могут существенным образом влиять на транспортные свойства многослойной структуры, затрудняет интерпретацию явления.

В связи с этим в настоящей работе проведено исследование характера изменения потенциального барьера в структуре металл/полупроводник вблизи температуры фазового перехода II рода в металле.

\section{2. Методика эксперимента}

В работе была исследована барьерная структура $\mathrm{Cr} / \mathrm{Si}$. В качестве второго электрода было использовано золото. Хром был выбран в связи с наличием у него фазового перехода антиферромагнетик-парамагнетик, происходящим при $T_{c}=310 \mathrm{~K}$. Хром был в виде монокристалла размером $5 \times 5$ мм. Коммерческий кремний $p$-типа $(100 К Д Б 12(100) \pm 0.5$ толщина $450 \pm 10$ мкм). Кремний был в виде пластин с высококачественной полированной поверхностью. Шероховатость поверхности, оцененная с помощью метода атомно-силовой микроскопии, составила $R_{a}=1 \mathrm{Hм}$.

Золотой электрод наносился на поверхность кремниевой пластины методом термодиффузионного осаждения в вакууме. Электрический контакт с хромовым электродом осуществлялся с помощью прижимного контакта. Экспериментальные структуры размещались на термостолике вакуумного азотного криостата, который позволял стабилизировать температуру и производить линейный нагрев и охлаждение в интервале от 90 до $573 \mathrm{~K}$. 
В ходе экспериментов были проведены два основных вида измерений. Это измерение температурной зависимости проводимости. Вторая группа измерения была связана с измерением вольт-амперных характеристик $(\mathrm{BAX})$ в зависимости от температуры образцов. Измерения ВАХ проводились в интервале температур от 223 до $373 \mathrm{~K}$ (с шагом по температуре $10 \mathrm{~K}$ ) с помощью источника-измерителя Keithley-2400.

\section{3. Экспериментальные результаты и обсуждение}

На рис. 1 приведена температурная зависимость проводимости структуры $\mathrm{Cr} / \mathrm{Si} / \mathrm{Au}$. При температуре $T \sim 223 \mathrm{~K}$ проводимость структуры минимальная и составляет $\sigma \sim 3.35 \cdot 10^{-8} \mathrm{CM}$. С ростом температуры наблюдается небольшой рост проводимости, и при темпеpaтуре $T \sim 273 \mathrm{~K}$ она составляет $\sigma \sim 7.46 \cdot 10^{-8}$ См. Достижение температуры $T \sim 293 \mathrm{~K}$ характеризуется резким скачкообразным увеличением проводимости структуры, которая достигает максимального в данном измерении значения $\sigma \sim 7.85 \cdot 10^{-6} \mathrm{CM}$.

Дальнейшее увеличение температуры приводит к тому, что проводимость структуры так же скачком уменьшается и при $T \sim 333 \mathrm{~K}$ составляет $\sigma \sim 1.07 \cdot 10^{-7} \mathrm{CM}$. Далее при нагреве до $T \sim 373 \mathrm{~K}$ на зависимости не наблюдается каких-либо резких изменений проводимости и при данной температуре она составляет $\sigma \sim 1.1 \cdot 10^{-7} \mathrm{CM}$.

Изменение проводимости вблизи температуры фазового перехода $\mathrm{Cr}$ может быть связано с изменением параметров потенциального барьера на границе раздела

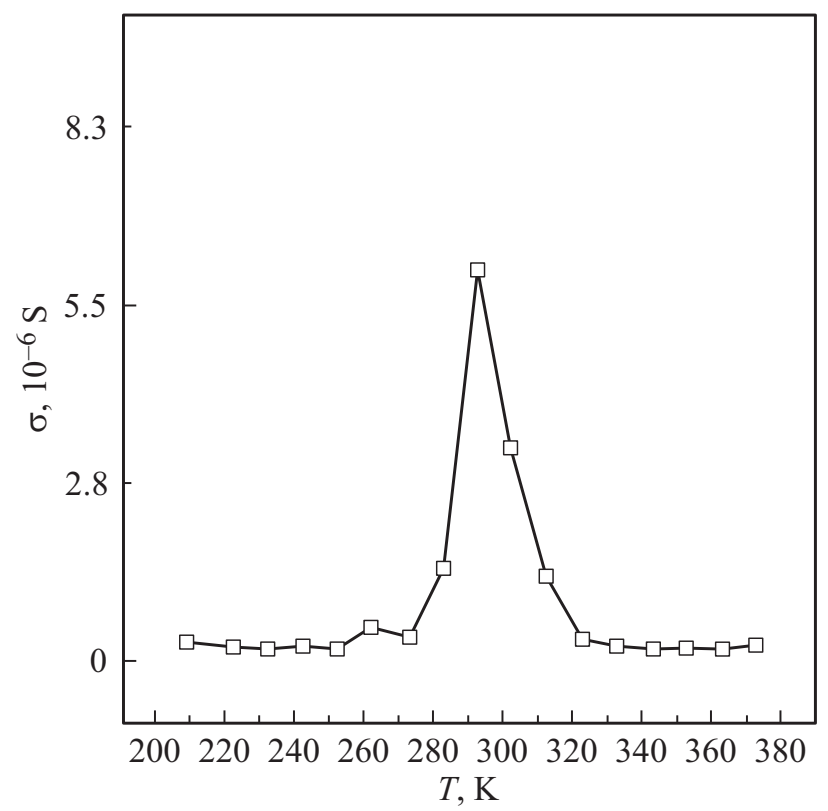

Рис. 1. Температурная зависимость проводимости в структуре $\mathrm{Au} / \mathrm{Si} / \mathrm{Cr}$ вблизи области фазового перехода антиферромагнетик-парамагнетик в $\mathrm{Cr}$.

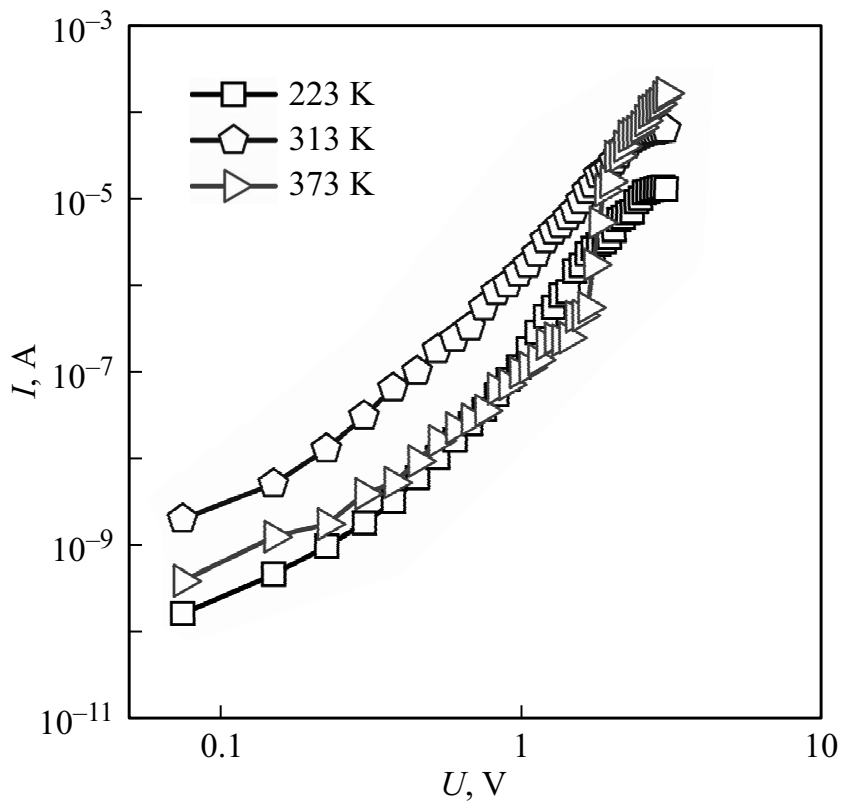

Рис. 2. Вольт-амперные характеристики структуры $\mathrm{Cr} / \mathrm{Si} / \mathrm{Au}$, измеренные при различных температурах.

$\mathrm{Cr} / \mathrm{Si}$ вследствие изменения положения уровня Ферми $\mathrm{Cr}$ в результате фазового перехода.

Экспериментальная оценка изменения высоты потенциального барьера была сделана на основе анализа BAX структуры Cr/Si/Au. На рис. 2 представлены BAX, измеренные при различных температурах до и после фазового перехода в $\mathrm{Cr}$.

Для анализа этих зависимостей мы использовали формализм модели барьера Шоттки. В рамках этой модели идеальная ВАХ контакта Шоттки будет описываться выражением [1]

$$
J=A^{*} T^{2} \exp \left(-\frac{q \varphi_{B 0}}{k T}\right) \exp \left(\frac{q(\Delta \varphi+V)}{k T}\right),
$$

где $\varphi_{B 0}$ - асимптотическое значение высоты потенциального барьера при нулевом поле, $A^{*}$ - эффективная постоянная Ричардсона, $\Delta \varphi-$ понижение барьера за счет эффекта Шоттки, $V$ - приложенное напряжение, $q$ - заряд, $k$ - постоянная Больцмана и $T-$ температура.

Высоту барьера можно получить из формулы

$$
\varphi_{B 0}=\frac{k T}{q} \ln \left(\frac{A^{*} T^{2}}{J_{s}}\right)
$$

$J_{s}$ - плотность тока насыщения, которая находится путем линейной экстраполяции ВАХ к $V=0$. Исходя из уравнения (2) изменение потенциального барьера до и после фазового перехода можно оценить из выражения

$$
\Delta \varphi_{B 0}=\frac{k}{q}\left(T_{1}-T_{2}\right) \ln \left(\frac{T_{1}^{2} J_{s 2}}{T_{2}^{2} J_{s 1}}\right) .
$$


На рис. 3 представлены ВАХ в полулогарифмических координатах, измеренные в структуре $\mathrm{Cu} / \mathrm{Si} / \mathrm{Cr}$ до и после фазового перехода в хроме, при температурах $T_{1}=293$ и $T_{2}=373 \mathrm{~K}$.

Плотность тока насыщения для $T_{1}=293 \mathrm{~K}$ составляет $J_{s 1}=8.3 \cdot 10^{-6} \mathrm{~A} / \mathrm{cm}^{2}, \quad$ а для $T_{2}=333 \mathrm{~K}$ $J_{s 2}=8.94 \cdot 10^{-9} \mathrm{~A} / \mathrm{cm}^{2}$. Таким образом, из выражения (3) следует, что разница в высоте потенциального барьера контакта $\mathrm{Cr} / \mathrm{Si}$ до и после фазового перехода составляет $\Delta \varphi_{B n}=0.24$ эВ. Таким образом установлено, что вблизи температуры фазового перехода в хроме происходит изменение высоты потенциального барьера.

Однако при использовании формулы (3) существует неопределенность в оценке площади контакта, так как контакт грани монокристалла хрома с поверхностью кремния являлся прижимным. В связи с этим полученное значение относительного изменения потенциального барьера может оказаться неточным.

Поэтому нами дополнительно была проведена оценка $\Delta \varphi$ на основе другого подхода. Известно [1], что для исследования поверхностей раздела, где электрически активная площадь может составлять только часть геометрической площади, для определения параметров контакта применяют метод энергии активации. Суть данного метода заключается в перестроении температурных зависимостей тока при постоянном приложенном напряжении смещения в координатах $\ln \left(I / T^{2}\right)$ от $1 / T$. Высота потенциального барьера, согласно выражению (4)

$$
\ln \left(\frac{I}{T^{2}}\right)=\ln \left(S A^{*}\right)-\frac{q\left(\varphi_{B n}-V\right)}{k T},
$$

будет определяться тангенсом угла наклона. Здесь $S-$ площадь электрически активной области. Произведение

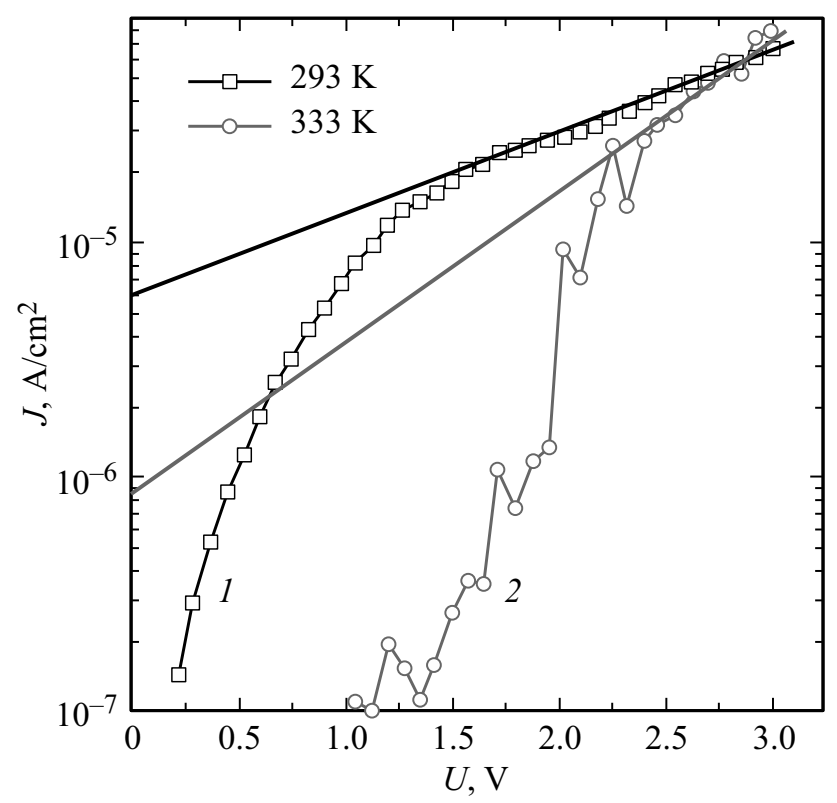

Рис. 3. Вольт-амперные характеристики структуры $\mathrm{Au} / \mathrm{Si} / \mathrm{Cr}$, представленные в полулогарифмических координатах $\ln (J)=f(U)$. Обозначения на кривых соответствуют: $1-293$, $2-333 \mathrm{~K}$.

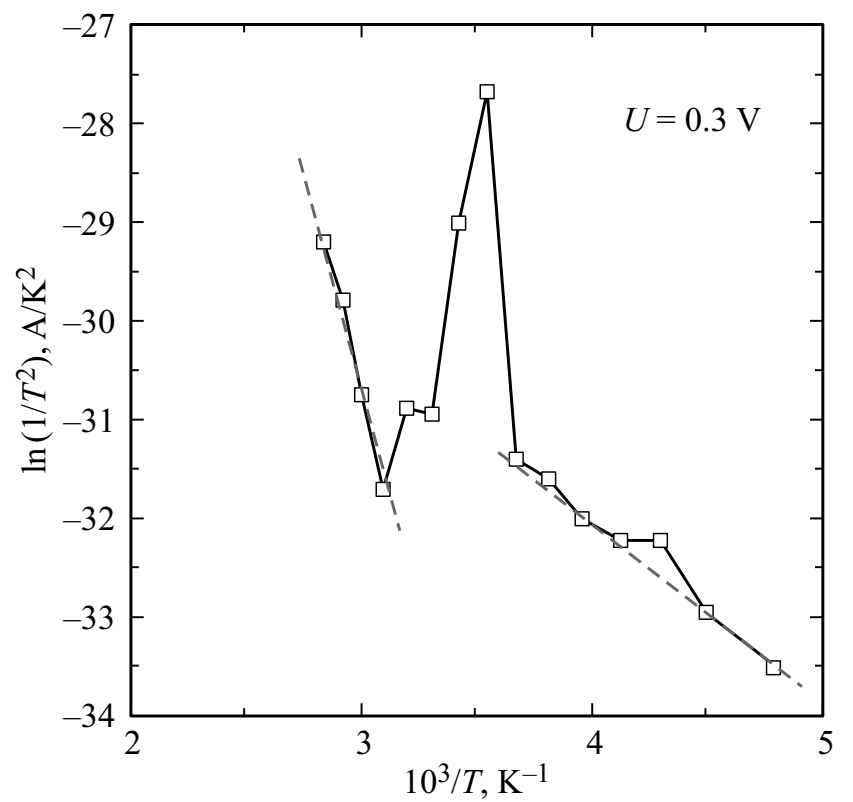

Рис. 4. Зависимость параметра $\ln \left(I / T^{2}\right)$ от $1 / T$ для структуры $\mathrm{Cr} / \mathrm{Si} / \mathrm{Au}$.

электрически активной площади $\mathrm{S}$ на эффективную постоянную Ричардсона находится из линейной экстраполяции зависимости до $1 / T=0$.

На рис. 4 приведена зависимость $\ln \left(I / T^{2}\right)=f(1 / T)$, измеренная в структуре $\mathrm{Au} / p-\mathrm{Si} / \mathrm{Cr}$, при напряжении, равном $0.3 \mathrm{~B}$.

На полученной зависимости выделяются две области, разделенные температурой, соответствующей температуре фазового перехода в хроме. В каждой из этих областей можно построить линейную аппроксимацию с разными углами наклона к осям координат. Можно сделать вывод о разной величине потенциального барьера до и после температуры фазового перехода. Изменение высоты потенциального барьера, определенное из тангенса угла наклона для двух прямолинейных участков, составляет $\Delta \varphi_{B n} \sim 0.18$ эВ. Данная величина находится в хорошем согласии со значением, определенным из уравнения (3), но на 0.06 эВ меньше. В принципе это означает, что оба метода дают адекватные значения величины потенциальных барьеров, но второй метод должен быть точнее, так как при его использовании нет необходимости в определении площади контакта.

\section{4. Заключение}

Таким образом, полученные результаты исследования транспортных свойств структуры $\mathrm{Au} / \mathrm{Si} / \mathrm{Cr}$ в области фазового перехода 2-го рода в $\mathrm{Cr}$ типа антиферромагнетик/парамагнетик показали, что особенности в проводимости обусловлены изменением потенциального барьера на контакте $\mathrm{Si} / \mathrm{Cr}$, вызванным разным положением уровня Ферми в хроме для антиферромагнитного и парамагнитного состояний. 


\title{
Список литературы
}

[1] С. Зи. Физика полупроводниковых приборов (М., Мир, 1984) кн. 1.

[2] М. Ламперт, П. Марк. Инжекционные токи в твердых mелах (М., Мир, 1973).

[3] M. Matlak, M. Pietruszka. J. Alloys Comp., 291, 21 (1999).

[4] В.М. Корнилов, А.Н. Лачинов. Письма ЖЭТФ, 61 (11), 902 (1995).

[5] M. Matlak, M. Pietruszka. Phys. Status Solidi B, 241 (1), 163 (2004).

Редактор Г.А. Оганесян

\section{Effect of a second-order phase transition on electrical conductivity metal/semiconductor structures}

I.R. Nabiullin ${ }^{1}$, R.M. Gadiev' ${ }^{1}$, A.N. Lachinov²

${ }^{1}$ Bashkir State Pedagogical University named after M. Akmulla,

450000 Ufa, Russia

${ }^{2}$ Institute of Physics of Molecules and Crystals, Ufa Scientific Center Russian Academy of Sciences, 450054 Ufa, Russia

\begin{abstract}
The paper presents the results of a study of the properties of the potential $\mathrm{Cr}-p$-Si barrier near the temperatures of the phase transition of an antiferromagnetic/paramagnetic in $\mathrm{Cr}$. A significant change in the potential barrier and an anomalous increase in conductivity in the $\mathrm{Cr}-p$ - $\mathrm{Si}-\mathrm{Au}$ structure near the phase transition temperature of the 2nd kind of antiferromagnetic/paramagnet in chromium was found. It has been established that as the phase transition temperature approaches the structure, an increase in current fluctuations is observed in the structure. Interpretation of the experimental results obtained is based on the assumption that the observed changes in the electron transport properties of the $\mathrm{Cr}-\mathrm{Si}$ contact are due to a change in the position of the Fermi-chromium quasi-level because of a secondorder phase transition.
\end{abstract}

